\title{
Re: Universal screening versus risk-based protocols for antibiotic prophylaxis during childbirth to prevent early-onset Group B streptococcal disease: A systematic review and meta-analysis. (First comment on BJOG-19-0865.R2)
}

\author{
Farah Seedat ${ }^{1}$ and John Marshall ${ }^{1}$ \\ ${ }^{1}$ UK National Screening Committee
}

May 5, 2020

Letter to the Editor, BJOG Exchange

Re: Universal screening versus risk-based protocols for antibiotic prophylaxis during childbirth to prevent early-onset Group B streptococcal disease: A systematic review and meta-analysis

Dear Editor,

We read the systematic review and meta-analysis "Universal screening versus risk-based protocols for antibiotic prophylaxis during childbirth to prevent early-onset Group B Streptococcal disease" with great interest. ${ }^{1}$ This systematic review correctly identified that overtreatment would occur in both risk-based and universal screening strategies. While our review, ${ }^{2}$ cited by the authors, only estimated the over-detection and potential overtreatment in a screening strategy and did not compare this to the amount of overtreatment in a risk-based strategy, we would like to make a few points about this comparison.

The authors found that there was no difference in the percentage of women receiving IAP in risk-based or screening protocols (29\% versus $31 \%$ respectively). However, the impact of screening on the rate of treatment exposure may depend on the details of the prevention strategies. It is important to ask what GBS risk factors are included in a risk-based strategy and, whether pregnant women with these risk factors, would be offered screening for GBS carriage and offered IAP if they have a negative GBS screening result in a universal screening strategy.

In the UK context, an expert panel agreed that universal screening would be implemented in addition to risk-based prevention and not as a replacement. ${ }^{3}$ Consequently, in the UK, screening may do little to reduce IAP in women who have GBS risk factors, but it would introduce IAP in a group of predominantly low risk women who carry GBS at term but most of whom would not have a neonate with EOGBS in the absence of IAP. Adding screening to risk-based prevention, a model in the UK estimated that screening would result in 96,260 pregnant women offered IAP treatment in addition to those already treated within the risk-based strategy alone. ${ }^{3}$

It is the balance of the overtreatment and potential harm against the benefits from screening in this group of pregnant women that is crucial. The lack of data on the clinically relevant outcomes in babies with EOGBS born to these pregnant women and the lack of data on the long-term outcomes of IAP treatment in this group hampers the assessment on both sides of this equation. ${ }^{2}$

We agree with the authors that clinical data are needed to verify whether screening would increase or decrease the amount of IAP exposure compared with risk-based prevention. We are hopeful that the upcoming NIHR 
HTA trial of screening for maternal GBS carriage will shed light on this in the UK context. ${ }^{4}$ With respect to screening itself, clinical data on a range of issues are needed to judge the impact of this intervention. Data are needed on the long-term outcomes of EOGBS disease in term births, and the long-term outcomes of IAP treatment in women with GBS carriage who are at low risk of having a baby with EOGBS. In addition, choice of place of birth has been identified as an important element of obstetric care so the impact of treatment on the experience of birth is another factor that may need to be considered. Without data on such issues, it is very difficult to determine that the benefit of universal screening outweighs the harm.

Farah Seedat \& John Marshall

UK National Screening Committee Evidence Team, Floor 5, Wellington House, 133-155 Waterloo Road, London, SE1 8UG.

\section{References}

1. Hasperhoven GF, Al-Nasiry S, Bekker V, et al. Universal screening versus risk-based protocols for antibiotic prophylaxis during childbirth to prevent early-onset Group B streptococcal disease: a systematic review and meta-analysis. BJOG: An International Journal of Obstetrics 83 Gynaecology 2020;n/a(n/a) doi: 10.1111/1471-0528.16085

2. Seedat F, Geppert J, Stinton C, et al. Universal antenatal screening for group B streptococcus may cause more harm than good. 2019;364:1463. doi: 10.1136/bmj.1463 \%J BMJ

3. Bevan D, White A, Marshall J, et al. Modelling the effect of the introduction of antenatal screening for group B Streptococcus (GBS) carriage in the UK. BMJ open 2019;9(3):e024324-e24. doi: 10.1136/bmjopen2018-024324

4. National Institute for Health Research. The clinical and cost-effectiveness of testing for Group B Streptococcus: a cluster randomised trial with economic and acceptability evaluations (GBS3). United Kingdom: National Institute for Health Research; 2019 [Available from: https://fundingawards.nihr.ac.uk/award/17/86/06 accessed 14 February 2020. 